\title{
First-Line Ablation of Ventricular Tachycardia in Ischemic Cardiomyopathy: Stratification of Outcomes by Left Ventricular Function
}

\author{
David Briceno ${ }^{1}$, Jorge Romero ${ }^{2}$, Kavisha Patel ${ }^{1}$, Andy Zhang ${ }^{1}$, Isabella Alviz ${ }^{3}$, Ruike \\ Yang $^{4}$, Daniel Rodriguez ${ }^{3}$, Dhanunjaya Lakkireddy ${ }^{5}$, Domenico Della Rocca ${ }^{6}$, Nicola \\ Tarantino $^{7}$, rakesh gopinathannair ${ }^{5}$, Andrea Natale ${ }^{8}$, and Luigi Di Biase ${ }^{9}$ \\ ${ }^{1}$ Montefiore Hospital and Medical Center \\ ${ }^{2}$ Brigham and Women's Hospital/Harvard Medical School. \\ ${ }^{3}$ Montefiore Medical Center \\ ${ }^{4}$ Henan Provincial People's Hospital \\ ${ }^{5}$ The Kansas City Heart Rhythm Institute (KCHRI) @ HCA MidWest \\ ${ }^{6}$ University Hospital of Rome \\ ${ }^{7}$ Universita degli Studi di Foggia \\ ${ }^{8}$ St. David's Medical Center \\ ${ }^{9}$ Texas Cardiac Arrhythmia Institute at St. David's Medical Center
}

July 1,2020

\begin{abstract}
Introduction: Randomized controlled trials (RCTs) have shown improved outcomes in patients undergoing first-line catheter ablation of ventricular tachycardia/ventricular fibrillation (VT/VF) in patients with ischemic cardiomyopathy (ICM). Herein, outcomes were stratified based on left ventricular ejection fraction (LVEF). Methods: RCTs evaluating first-line ablation versus medical therapy in patients with VT and ICM were included. Risk estimates and $95 \%$ confidence intervals (CI) were measured. Results: Four RCTs with a total of 505 patients (mean age $66 \pm 9$ years, $89 \%$ male, $80 \%$ with previous revascularization) were included. Mean LVEF was $35 \pm 8 \%$. At a mean follow-up of $24 \pm 9$ months, a significant benefit in survival-free from appropriate ICD therapies was observed in all patients undergoing first-line catheter ablation compared to medical management (RR 0.70, 95\% CI 0.56-0.86). In patients with moderately depressed LVEF (>30-50\%), first line VT ablation was associated with a statistically significant reduction in the composite endpoint of survival-free VT/VF and appropriate ICD therapies (HR: 0.52, 95\% CI: 0.36-0.76), whereas there was no difference in patients with severely depressed LVEF (30-50\%).
\end{abstract}

\section{INTRODUCTION}

In patients with ischemic cardiomyopathy (ICM), ventricular tachycardia and ventricular fibrillation $(\mathrm{VT} / \mathrm{VF})$ typically originate from a relatively discrete portion of the myocardium, within or bordering the infarct zone that is amenable to catheter ablation. ${ }^{1}$ However, the mainstay of treatment for these patients is typically based on medical therapy and implantable cardioverter defibrillators (ICDs). ${ }^{2}$ Use of anti-arrhythmic drugs (AAD) is typically the first approach as an adjunctive therapy to reduce ICD therapies; yet, success is limited and may be associated with significant drug-related adverse events. ${ }^{3}$ Conversely, catheter ablation has emerged as a an important therapeutic strategy for VT/VF with the advent of improved mapping technologies and ablation strategies, particularly the success seen with substrate based approaches. ${ }^{4}$ As such, 
randomized controlled trials (RCTs) have shown improved outcomes in patients undergoing first-line catheter ablation for VT in patients with ICM. ${ }^{5,6}$ However, increased early mortality has been reported in patients with severely depressed left ventricular ejection fraction (LVEF), whereas outcomes in moderately depressed LVEF seem different. ${ }^{7}$ Herein, we characterized outcomes based on LVEF of patients with ICM presenting with VT undergoing first line VT ablation compared to an initial approach based on medical therapy.

\section{METHODS}

\section{Search strategy}

We searched PubMed, Embase, and Cochrane Central Register of Clinical Trials (Cochrane Library, Issue 5, 2020) to identify studies evaluating the outcomes of patients with prior myocardial infarction that presenting with VT that were randomized to first-line therapy with catheter ablation or medical therapy. We used the terms: "ventricular tachycardia" OR "VT" OR "ventricular fibrillation" OR "VF" OR "ventricular tachyarrhythmia") AND ("ischemic cardiomyopathy" OR "ICM" OR "myocardial infarction" OR "MI") AND ("catheter ablation" OR "CA" OR "radiofrequency ablation"). Our search was restricted to human studies, published in peer-reviewed journals up to April 2020. No language restriction was applied. The reference lists of identified articles were also reviewed. We also searched clinicaltrials.gov to identify ongoing and unpublished trials.

\section{Selection criteria}

The Preferred Reporting Items for Systematic Reviews and Meta-Analyses (PRISMA) statement for reporting systematic reviews and meta-analyses was applied to the methods of this study. Studies with the following characteristics were considered eligible for this analysis: a) had a randomized controlled design; b) included patients with prior myocardial infarction and VT; c) studies with outcomes stratified by LVEF; d) included patients that were randomized to first line VT ablation or a control group based on medical therapy as an initial therapy; e) patients were followed for an average duration of at least 12 months; f) studies included survival-free from VT/VF and/or rates of appropriate ICD therapies as their endpoints.

\section{Study outcomes}

\section{Primary outcome}

The primary outcomes were: 1) all-cause mortality, 2) appropriate ICD therapies, and 3) adverse events in all the patients included.

\section{Secondary outcomes}

The secondary outcomes were: 1) composite of survival-free from VT/VF and appropriate ICD therapies in patients with moderately depressed LVEF (>30-50\%), and 2) composite of survival-free from VT/VF and appropriate ICD therapies in patients with severely depressed LVEF $(</=30 \%)$.

\section{Sensitivity Analysis}

Sensitivity analyses were performed for the secondary endpoints, considering the composite nature of the outcomes, using the leave-one-study-out method in order to address the influence of each study by testing whether deleting each individually would significantly change the pooled results of the meta-analysis. Additionally, chronological cumulative analyses were used to test whether the effect size and precision would shift based on technical advancement of mapping technology and ablation strategies.

\section{Data Extraction and Quality Assessment}

Two investigators (D.F.B and J.R) independently screened all titles, abstracts and manually searched the full text versions of all pertinent studies that fulfilled the inclusion criteria. References of the relevant studies were independently reviewed for potential identification of further studies. Two investigators (D.F.B and J.R) independently assessed the quality items. Disagreements were resolved by consensus. Quality assessment of all included studies was done by using the 6 domains of the Cochrane tool for assessing risk for bias of RCTs. 


\section{Statistical analysis}

Hazard ratios (HR) or Mantel-Haenszel (MH) risk ratio (RR) models where appropriate and 95\% confidence intervals (CI) were used to summarize data across treatment arms. The Cochran Q-test and the Higgins I-squared $\left(\mathrm{I}^{2}\right)$ statistic were used for heterogeneity testing; in cases of heterogeneity (defined as $\mathrm{I}^{2}>25 \%$ ), random effects models of DerSimonian and Laird were used, otherwise $\left(\mathrm{I}^{2}<25 \%\right)$ fixed effects models were used. To address publication bias, we used the funnel plots. If any bias was observed, further bias quantification was measured using the Begg-Mazumdar test, Egger test, and the Duval and Tweedie's trim and fill test.

Descriptive statistics are presented as means and standard deviations (SD) for continuous variables or number of cases (n) and percentages (\%) for dichotomous and categorical variables. Number needed to treat (NNT) was calculated. Statistical analysis was performed in line with recommendations from the Cochrane Collaboration and the PRISMA guidelines, using Review Manager (RevMan) [Computer program]. Version 5.3. Copenhagen: The Nordic Cochrane Centre, The Cochrane Collaboration, 2014, and the Comprehensive Meta-Analysis Software version 2.0 (Biostat, Inc).

\section{RESULTS}

\section{Qualitative Analysis}

\section{Study selection}

We screened 455 abstracts, out of which 12 full-text articles were retrieved and reviewed for possible inclusion based on inclusion criteria. Ultimately, four studies fulfilled the inclusion criteria and were included in the analysis. Flow chart of the literature review is illustrated in Figure 1 .

\section{Baseline characteristics}

The baseline characteristics of the patients included in this meta-analysis are reported in Table $\mathbf{1}$. A total of 505 patients (mean age $66 \pm 9$ years, $89 \%$ males) were analyzed. The mean duration of follow-up was $24 \pm 9$ months. Previous revascularization with percutaneous transluminal coronary angioplasty (PTCA) or coronary artery bypass grafting $(\mathrm{CABG})$ was performed in $80 \%$ of patients. Mean duration since last MI was $10 \pm 9$ years. Mean LVEF was $35 \pm 8 \%$, and $84 \%$ of patients were on beta-blockers. Table 2 illustrates a summary of the included studies.

\section{Quantitative Analysis}

\section{Primary endpoints}

A significant benefit was observed with first-line catheter ablation compared to medical management in survival-free from appropriate ICD therapies, with a $26 \%$ relative risk reduction and $14 \%$ absolute risk reduction when compared with the medical treatment group (RR 0.70, 95\% CI 0.56-0.86) (Figure 2 ). There was no difference in all-cause mortality (RR 0.98, 95\% CI 0.52-1.82) and in adverse events between both treatment strategies (RR 1.51, 95\% CI 0.89-2.54) (Supplemental Figure 1 ).

\section{Secondary endpoints}

In patients with moderately depressed LVEF ( $>30-50 \%)$, survival-free from VT/VF in patients who underwent first-line catheter ablation was $56 \%$ vs. $45 \%$ in those receiving conservative treatment. A significant benefit of survival-free from $\mathrm{VT} / \mathrm{VF}$ was observed with first-line catheter ablation, with a $24 \%$ relative risk reduction and $11 \%$ absolute risk reduction when compared with the medical treatment group (HR: 0.56, 95\% CI: 0.38-0.83) (Figure 2 ).

First line VT ablation was associated with a statistically significant reduction in the composite endpoint of survival-free VT/VF and appropriate ICD therapies in patients with moderately reduced LVEF (HR: 0.52, 95\% CI: 0.36-0.76) (Figure 3) . In patients with severely depressed LVEF $(</=30 \%)$, first line VT ablation 
was not associated with a reduction in the composite endpoint of survival-free VT/VF and appropriate ICD therapies (HR: 0.56, 95\% CI: 0.24-1.32)(Figure 3) .

\section{Sensitivity analysis}

Sensitivity analysis for the secondary endpoints involving the removal of each of the studies sequentially demonstrated that if some of the studies were removed from the analysis, the summary risk estimates for the composite survival-free VT/VF and appropriate ICD therapies was not influenced making the overall results significant (Figure 4 ). The chronologic cumulative analysis for each outcome before inclusion of all studies in the final effect summary did not have a significant impact in the overall final effect (oldest RCT from 2007 and most recent in 2020) (Figure 4 ).

\section{Number needed to treat}

The absolute difference in event rates yielded an NNT of 7 and 10 patients in order to improve survivalfree from appropriate ICD therapies in all patients and survival-free from VT/VF in those with moderately depressed LVEF, respectively.

\section{Quality assessment and publication bias}

Funnel plots did not show asymmetry, suggesting lack of bias for any outcome (Supplemental Figure 2 ). A graph and summary of the tools recommended by the Cochrane Collaboration for the risk of bias (selection bias, performance bias, detection bias, attrition bias, reporting bias and other bias) identified in each individual RCT is shown in Figure 5 .

\section{DISCUSSION}

In patients with ICM, VT/VF is a major cause of morbidity and mortality. Thus, strategies to minimize arrhythmia burden in these patients is essential. Our study, which included 505 patients with a mean followup duration of $24 \pm 9$ months, showed that patients with ICM presenting with VT/VF, first-line catheter ablation have a significant lower rate of recurrent appropriate ICD therapies compared to patients receiving an initial approach based on medical therapy (RR 0.70, 95\% CI 0.56-0.86). This was significantly important in the subgroup of patients with moderately depressed LVEF (>30-50\%) (HR: 0.52, 95\% CI: 0.36-0.76) compared to patients with severely depressed LVEF $(</=30 \%)$, where no benefit was seen (HR: $0.56,95 \%$ CI: 0.24-1.32).

In patients implanted with secondary prevention ICDs, shocks have been reported in up to $39 \%$ of patients within the first year of implantation. ${ }^{3}$ Recurrent ICD shocks, may cause deterioration of heart failure, increase hospitalization rates, and have been associated with increased mortality. ${ }^{8}$ The SCD-HeFT trial noted that a single appropriate ICD shock increased patient mortality-risk up to five-fold. ${ }^{8}$ Shocks with their potential to worsen HF, also tend to alter mode of mortality in the direction of non-arrhythmic death, thereby, offsetting the arrhythmic mortality advantage offered by ICDs. ${ }^{9}$ Moreover, they do not alter the underlying pathological substrate, and do not prevent VT. Therefore, it is important to adopt a strategy that reduces the absolute incidence of VT/VF, and as such, subsequent ICD shocks. Utilization of AADs and ICD reprogramming have been employed with moderate degrees of success. ${ }^{3}$ Accordingly, the VANISH trial demonstrated that there was a significantly lower rate of the composite primary outcome of death, VT storm, or appropriate ICD shock among patients undergoing catheter ablation than among those receiving an escalation in AAD (HR, $0.72 ; 95 \%$ CI, 0.53 to $0.98 ; \mathrm{p}=0.04) \cdot{ }^{10}$

In patients with ICM, VT usually arises from a fairly distinct region of the myocardium, within or neighboring the scar tissue. This scar tissue is comprised of non-excitable fibrous material, with islands of surviving myocytes composing the substrate for VT. A general physiology seems to be common in patients with ICM and sustained monomorphic VT, characterized by larger endocardial lower voltage zones, more frequent fractionated, very late voltage potentials as compared to patients with a similar profile without VT. ${ }^{11}$ Nonetheless, scars are not homogenous considering tissue characteristics can be variable in each patient depending largely on the infarct type (i.e., size, location, revascularization time, etc.) posing a challenge 
for the treatment of VT/VF. Therefore, is not surprising that ablation studies have shown variable results reflecting conservative guideline recommendations. Guidelines recommend VT ablation as a Class I indication in patients with prior MI, only with recurrent episodes of symptomatic sustained VT, or VT storm, and have failed or are intolerant to AADs. ${ }^{2}$ However, this recommendation may not apply to every patient, as there seems to be a significant benefit in different subgroups of patients including those undergoing early ablation and preserved LVEF. ${ }^{12}$ In general, a lower threshold to consider catheter ablation of VT (whether it is determined by failure of 1 AAD or immediately after a VT episode) could be associated with a favorable outcome. ${ }^{12}$ Furthermore, data from the International VT Ablation Center Collaborative Group, reported higher rates of both VT recurrence and mortality in patients with lower EF and higher NYHA status. ${ }^{13}$ In contrast, ICM and higher EF $(>30 \%)$ were associated with lower probability of VT recurrence that was reflected in improved transplant-free survival compared to those with VT recurrence (93\% vs. 89\%, adjusted HR $3.190(1.517-6.707) ; \mathrm{p}=0.002) .{ }^{13}$

Four randomized controlled trials have assessed the impact of first-line VT catheter ablation in the setting of ICM. ${ }^{5,6,14,15}$ SMASH VT and VTACH trials showed improved outcomes with first-line VT ablation in patients with ICM presenting with VT. ${ }^{5,6}$ However, the SMS and the recently published BERLIN VT trial, showed opposite results. ${ }^{14,15}$ As expected, all of these trials had methodological differences but the major approach studied is homogenous, considering the use of first-line VT ablation in each of them and a medication-based approach in the control groups. In terms of the control groups, AADs were used in $35 \%$ of patients in the VTACH study, $32 \%$ in SMS, and 33\% in BERLIN VT; however, only beta-blockers were used in SMASH VT. ${ }^{5,6,15,16}$ All control groups were medications based except in the BERLIN VT trial, where patients were randomized to receive ablation after the third appropriate ICD shock. ${ }^{15}$ Ultimately, 10 patients $(12 \%)$ received ablation in this group, which is perhaps one of the reasons the trial showed no benefit in the ablation groupo. ${ }^{15}$ They showed that first-line VT ablation did not reduce the combined endpoint of mortality or hospitalization for arrhythmia or worsening heart failure during 1 year of follow-up when compared to the deferred ablation strategy (HR, 1.09; 95\% CI, 0.62-1.92; $\mathrm{P}=0.77) .{ }^{15}$ Perhaps the study power, patient crossover, and endocardial-only ablation strategy were not enough to demonstrate a significant clinical benefit of ablation. VT circuits in ICM are generally thought to have a subendocardial location, easily accessible, and targeted with endocardial mapping and ablation. ${ }^{17}$ However, $34-75 \%$ of ICM patients may exhibit epicardial substrate, as confirmed by magnetic resonance imaging in post-infarct animal models. ${ }^{18}$ This may explain why a combined endocardial-epicardial ablation have been shown to be beneficial in ICM. We recently published a meta-analysis with 17 studies including 975 patients. ${ }^{19}$ After a mean follow-up of 27 months, endocardial-epicardial ablation was associated with a $35 \%$ reduction in risk of VT recurrence compared with endocardial ablation alone (RR: $0.65 ; 95 \%$ CI: 0.55 to $0.78 ; \mathrm{p}<0.001$ ). Sensitivity analysis showed lower risk of VT recurrence in ICM (RR: $0.43 ; 95 \%$ CI: 0.28 to $0.67 ; \mathrm{p}=0.0002)$ with a nonsignificant trend in NICM (RR: 0.87; 95\% CI: 0.70 to 1.08; $\mathrm{p}=0.20$ ). More importantly, an endocardial-epicardial approach was associated with reduced all-cause mortality (RR: $0.56 ; 95 \% \mathrm{CI}: 0.32$ to $0.97 ; \mathrm{p}=0.04$ ), particularly in patients with ICM. ${ }^{19}$ As such, perhaps early ablation using an endocardial-epicardial strategy in patients with LVEF >30-50\% could be the most beneficial therapeutic strategy.

Despite the mixed results from the four RCTs evaluating this concept, our results substantiate the use of VT ablation as a first-line approach in this population to improve survival-free from VT/VF, which may also have a positive impact from a healthcare resource utilization standpoint. Winterfield et al ${ }^{20}$, in a largescale, real-world, retrospective analysis of 523 patients noted a decline in median cardiac rhythm-related medical expenditures of approximately $\$ 5,400$ per patient-year $(\$ 7,318$ pre-ablation to $\$ 1,910$ post-ablation, $\mathrm{p}<0.0001)$. A significant reduction in all-cost total medical expenditure was noted in the subgroup of patients without repeat ablation $\left({ }^{\sim} 37 \%\right.$ reduction; $\left.\mathrm{p}=0.0005\right)$. The expenditures for device follow-ups were also noted to have a significant decline in this subgroup ( $\$ 906$ to $\$ 824, \mathrm{p}=0.05)$. Multiple clinical studies are currently underway aiming to address the best therapeutic approach of these patients: Does Timing of VT ablation Affect Prognosis in Patients with an ICD? (PARTITA) (NCT01547208); Pan-Asia United States PrEvention of Sudden Cardiac Death Catheter Ablation Trial (PAUSE-SCD) (NCT02848781); The Antiarrhythmics or Ablation for Ventricular Tachycardia 2 (VANISH 2) Study (NCT02830360). 


\section{Limitations}

Our meta-analysis has several limitations, which should be taken into account while interpreting the results. Only a small number of studies have assessed the impact of first-line VT ablation in patients with ICM, and the data available to stratify outcomes based on LVEF is limited; hence, the necessity to assess a composite endpoint. Also, techniques for VT mapping and ablation differed amongst the studies. Our results are applicable only to patients with ICM, and should not be generalized to NICM.

\section{CONCLUSION}

Patients with ICM presenting with VT undergoing first-line VT ablation have a significant lower rate of appropriate ICD therapies without a mortality difference compared to patients receiving an initial approach based on medical therapy. The beneficial effect in improved survival-free from VT/VF and appropriate ICD therapies was seen only in patients with moderately depressed LVEF. First-line VT ablation should be considered the therapeutic approach of choice in this selected group of patients to improve clinical outcomes.

\section{REFERENCES}

1. de Bakker JM, van Capelle FJ, Janse MJ, Wilde AA, Coronel R, Becker AE, Dingemans KP, van Hemel NM, Hauer RN. Reentry as a cause of ventricular tachycardia in patients with chronic ischemic heart disease: electrophysiologic and anatomic correlation. Circulation 1988;77:589-606.

2. Cronin EM, Bogun FM, Maury P, Peichl P, Chen M, Namboodiri N, Aguinaga L, Leite LR, Al-Khatib SM, Anter E, Berruezo A, Callans DJ, Chung MK, Cuculich P, d'Avila A, Deal BJ, Della Bella P, Deneke T, Dickfeld TM, Hadid C, Haqqani HM, Kay GN, Latchamsetty R, Marchlinski F, Miller JM, Nogami A, Patel AR, Pathak RK, Saenz Morales LC, Santangeli P, Sapp JL Jr, Sarkozy A, Soejima K, Stevenson WG, Tedrow UB, Tzou WS, Varma N, Zeppenfeld K. 2019 HRS/EHRA/APHRS/LAHRS expert consensus statement on catheter ablation of ventricular arrhythmias. Heart Rhythm 2020;17:e2-e154.

3. Connolly SJ, Dorian P, Roberts RS, Gent M, Bailin S, Fain ES, Thorpe K, Champagne J, Talajic M, Coutu B, Gronefeld GC, Hohnloser SH; Optimal Pharmacological Therapy in Cardioverter Defibrillator Patients (OPTIC) Investigators. Comparison of beta-blockers, amiodarone plus beta-blockers, or sotalol for prevention of shocks from implantable cardioverter defibrillators: the OPTIC Study: a randomized trial. JAMA 2006;295:165-171.

4. Briceño DF, Romero J, Villablanca PA, Londoño A, Diaz JC, Maraj I, Batul SA, Madan N, Patel J, Jagannath A, Mohanty S, Mohanty P, Gianni C, Della Rocca D, Sabri A, Kim SG, Natale A, Di Biase L. Long-term outcomes of different ablation strategies for ventricular tachycardia in patients with structural heart disease: systematic review and meta-analysis. Europace 2018;20:104-115.

5. Reddy VY, Reynolds MR, Neuzil P, Richardson AW, Taborsky M, Jongnarangsin K, Kralovec S, Sediva L, Ruskin JN, Josephson ME. Prophylactic catheter ablation for the prevention of defibrillator therapy. N Engl J Med 2007;357:2657-2665.

6. Kuck KH, Schaumann A, Eckardt L, Willems S, Ventura R, Delacrétaz E, Pitschner HF, Kautzner J, Schumacher B, Hansen PS; VTACH study group. Catheter ablation of stable ventricular tachycardia before defibrillator implantation in patients with coronary heart disease (VTACH): a multicentre randomised controlled trial. Lancet 2010;375:31-40.

7. Santangeli P, Frankel DS, Tung R, Vaseghi M, Sauer WH, Tzou WS, Mathuria N, Nakahara S, Dickfeldt TM, Lakkireddy D, Bunch TJ, Di Biase L, Natale A, Tholakanahalli V, Tedrow UB, Kumar S, Stevenson WG, Della Bella P, Shivkumar K, Marchlinski FE, Callans DJ; International VT Ablation Center Collaborative Group. Early Mortality After Catheter Ablation of Ventricular Tachycardia in Patients With Structural Heart Disease. J Am Coll Cardiol 2017;69:2105-2115.

8. Poole JE, Johnson GW, Hellkamp AS, et al. Prognostic importance of defibrillator shocks in patients with heart failure. N Engl J Med 2008;359:1009-1017. 
9. Hohnloser SH, Kuck KH, Dorian P, Roberts RS, Hampton JR, Hatala R, Fain E, Gent M, Connolly SJ; DINAMIT Investigators. Prophylactic use of an implantable cardioverter-defibrillator after acute myocardial infarction. N Engl J Med 2004;351:2481-2488.

10. Sapp JL, Wells GA, Parkash R, Stevenson WG, Blier L, Sarrazin JF, Thibault B, Rivard L, Gula L, Leong-Sit P, Essebag V, Nery PB, Tung SK, Raymond JM, Sterns LD, Veenhuyzen GD, Healey JS, Redfearn D, Roux JF, Tang AS. Ventricular Tachycardia Ablation versus Escalation of Antiarrhythmic Drugs. N Engl J Med 2016;375:111-121.

11. Haqqani HM, Kalman JM, Roberts-Thomson KC, Balasubramaniam RN, Rosso R, Snowdon RL, Sparks PB, Vohra JK, Morton JB. Fundamental differences in electrophysiologic and electroanatomic substrate between ischemic cardiomyopathy patients with and without clinical ventricular tachycardia. J Am Coll Cardiol 2009;54:166-173.

12. Romero J, Di Biase L, Diaz JC, Quispe R, Du X, Briceno D, Avendano R, Tedrow U, John RM, Michaud GF, Natale A, Stevenson WG, Kumar S. Early Versus Late Referral for Catheter Ablation of Ventricular Tachycardia in Patients With Structural Heart Disease: A Systematic Review and Meta-Analysis of Clinical Outcomes. JACC Clin Electrophysiol 2018;4:374-382.

13. Tung R, Vaseghi M, Frankel DS, Vergara P, Di Biase L, Nagashima K, Yu R, Vangala S, Tseng CH, Choi EK, Khurshid S, Patel M, Mathuria N, Nakahara S, Tzou WS, Sauer WH, Vakil K, Tedrow U, Burkhardt JD, Tholakanahalli VN, Saliaris A, Dickfeld T, Weiss JP, Bunch TJ, Reddy M, Kanmanthareddy A, Callans DJ, Lakkireddy D, Natale A, Marchlinski F, Stevenson WG, Della Bella P, Shivkumar K. Freedom from recurrent ventricular tachycardia after catheter ablation is associated with improved survival in patients with structural heart disease: An International VT Ablation Center Collaborative Group study. Heart Rhythm 2015; 12:1997-2007.

14. Kuck KH, Tilz RR, Deneke T, Hoffmann BA, Ventura R, Hansen PS, Zarse M, Hohnloser SH, Kautzner J, Willems S; SMS Investigators. Impact of Substrate Modification by Catheter Ablation on Implantable Cardioverter-Defibrillator Interventions in Patients With Unstable Ventricular Arrhythmias and Coronary Artery Disease: Results From the Multicenter Randomized Controlled SMS (Substrate Modification Study). Circ Arrhythm Electrophysiol 2017;10:e004422. doi: 10.1161/CIRCEP.116.004422.

15. Willems S, Tilz RR, Steven D, Kääb S, Wegscheider K, Gellér L, Meyer C, Heeger CH, Metzner A, Sinner MF, Schlüter M, Nordbeck P, Eckardt L, Bogossian H, Sultan A, Wenzel B, Kuck KH; BERLIN VT Investigators. Preventive or Deferred Ablation of Ventricular Tachycardia in Patients with Ischemic Cardiomyopathy and Implantable Defibrillator (BERLIN VT): A Multicenter Randomized Trial. Circulation 2020;141:1057-1067.

16. Dinov B, Fiedler L, Schönbauer R, Bollmann A, Rolf S, Piorkowski C, Hindricks G, Arya A. Outcomes in catheter ablation of ventricular tachycardia in dilated nonischemic cardiomyopathy compared with ischemic cardiomyopathy: results from the Prospective Heart Centre of Leipzig VT (HELP-VT) Study. Circulation 2014;129:728-736.

17. Tschabrunn CM, Roujol S, Nezafat R, Faulkner-Jones B, Buxton AE, Josephson ME, Anter E. A swine model of infarct-related reentrant ventricular tachycardia: Electroanatomic, magnetic resonance, and histopathological characterization. Heart Rhythm 2016;13:262-273.

18. Arenal A, Pérez-David E, Avila P, Fernández-Portales J, Crisóstomo V, Báez C, Jiménez-Candil J, RubioGuivernau JL, Ledesma-Carbayo MJ, Loughlin G, Bermejo J, Sánchez-Margallo FM, Fernández-Avilés F. Noninvasive identification of epicardial ventricular tachycardia substrate by magnetic resonance-based signal intensity mapping. Heart Rhythm 2014;11:1456-1464.

19. Romero J, Cerrud-Rodriguez RC, Di Biase L, Diaz JC, Alviz I, Grupposo V, Cerna L, Avendano R, Tedrow U, Natale A, Tung R, Kumar S. Combined Endocardial-Epicardial Versus Endocardial Catheter 
Ablation Alone for Ventricular Tachycardia in Structural Heart Disease: A Systematic Review and MetaAnalysis. JACC Clin Electrophysiol 2019;5:13-24.

20. Winterfield JR, Kent AR, Karst E, Dalal N, Mahapatra S, Bunch TJ, Reynolds MR, Wilber DJ. Impact of ventricular tachycardia ablation on health care utilization. Heart Rhythm 2018;15:355-362.

\section{FIGURE LEGENDS}

Figure 1. Flow chart of study selection

Figure 2. Survival-free from VT/VF and from appropriate ICD therapies in patients with ICM presenting with VT undergoing first-line catheter ablation compared to medical management. (A) Survival-free from appropriate ICD therapies in all patients; (B ) Survival-free from VT/VF in patients with moderately depressed LVEF (>30-50\%). ICD: implantable-cardioverter defibrillator; ICM: ischemic cardiomyopathy; VF: ventricular fibrillation; VT: ventricular tachycardia.

Figure 3. Forest plot reporting the hazard ratio illustrating the composite endpoint of survival-free VT/VF and appropriate ICD therapies in patients with ICM presenting with VT undergoing first-line catheter ablation compared to conservative treatment. (A ) Patients with moderately depressed LVEF ( $>30-50 \%)$; (B ) Patients with severely depressed LVEF $(</=30 \%)$. Diamond indicates overall summary estimates for the analysis: width of the diamond represents $95 \%$ CI; width of the shaded square represents the size of the population (fixed effects model was used in the analysis). CI: confidence interval; ICM: ischemic cardiomyopathy; VF: ventricular fibrillation; VT: ventricular tachycardia.

Figure 4. Forest plots reporting the sensitivity analysis for the secondary endpoint: (A) leave-one-study-out method for moderately depressed LVEF; (B) leave-one-study-out method for severely depressed LVEF; (C) Chronological cumulative analysis for moderately depressed LVEF; (D) Chronological cumulative analysis for severely depressed LVEF. Diamond indicates overall summary estimate for the analysis (width of the diamond represents the $95 \% \mathrm{CI}$ ); width of the shaded square, size of the population. $\mathrm{CI}=$ Confidence interval; ICD: implantable-cardioverter defibrillator; ICM: ischemic cardiomyopathy; LVEF: left ventricular ejection fraction; VF: ventricular fibrillation; VT: ventricular tachycardia.

Figure 5. (A) Methodological quality graph and (B) Methodological quality summary for the risk of bias from the included randomized controlled trials using the 6 domains of the Cochrane tool.

\section{Hosted file}

TABLES.docx available at https://authorea.com/users/338336/articles/464357-first-lineablation-of-ventricular-tachycardia-in-ischemic-cardiomyopathy-stratification-ofoutcomes-by-left-ventricular-function 


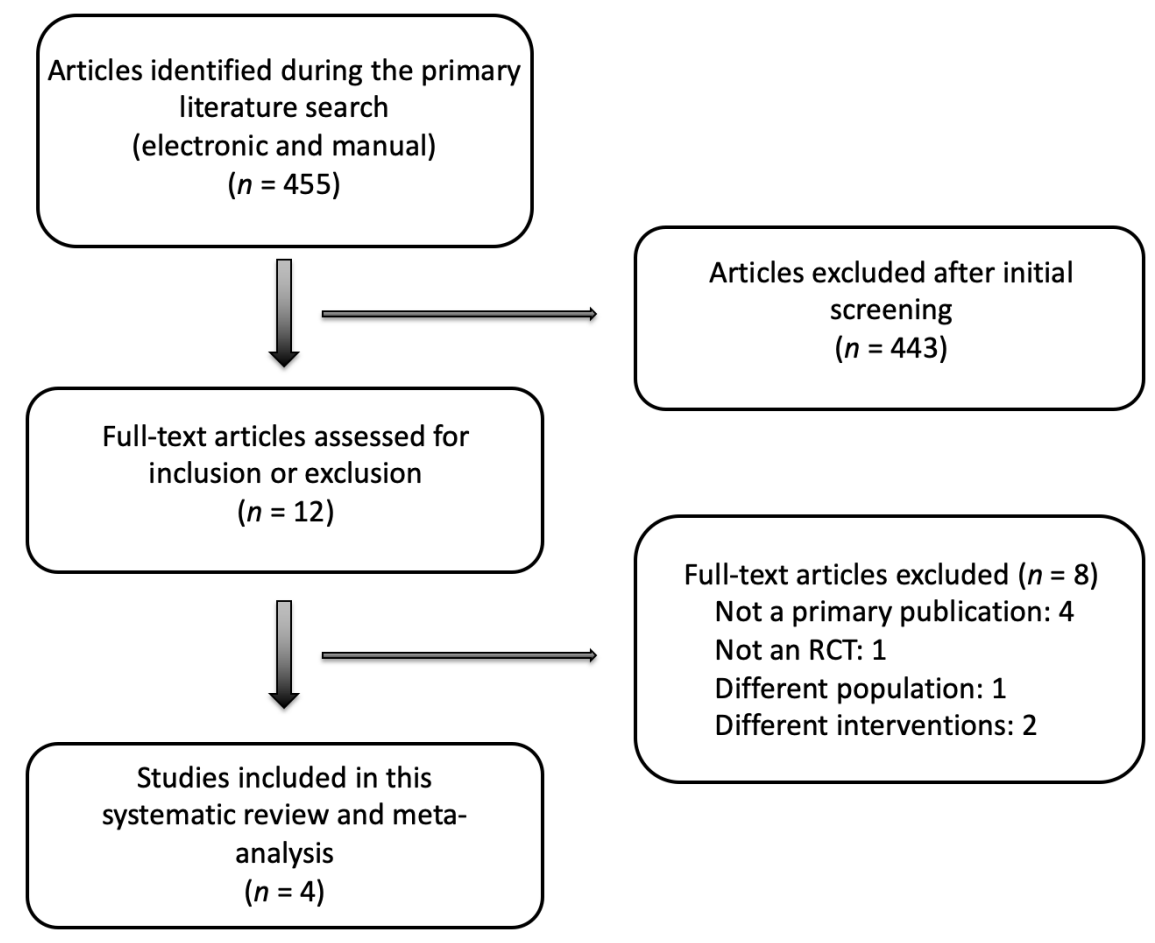

A

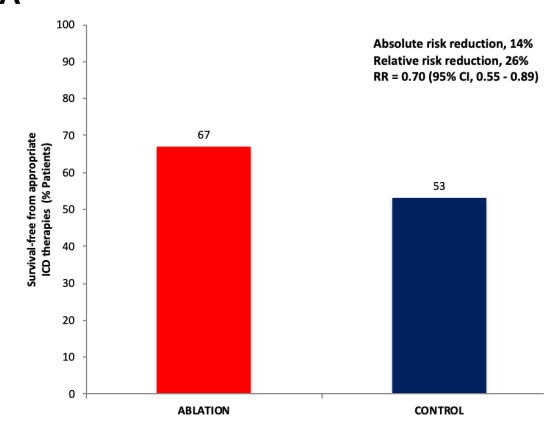

B

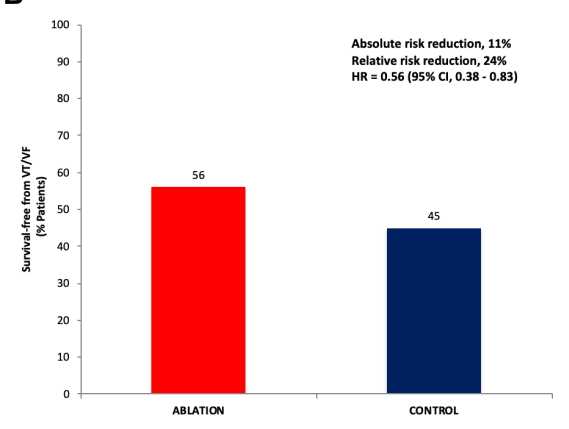


A

\begin{tabular}{|c|c|c|c|c|c|}
\hline Study name & Statisti & s for ea & h study & Hazard ratio and $95 \% \mathrm{Cl}$ & \\
\hline & $\begin{array}{c}\text { Hazard } \\
\text { ratio }\end{array}$ & $\begin{array}{c}\text { Lower } \\
\text { limit }\end{array}$ & $\begin{array}{l}\text { Upper } \\
\text { limit }\end{array}$ & & $\begin{array}{c}\text { Relative } \\
\text { weight }\end{array}$ \\
\hline Reddy 2007 & 0.18 & 0.04 & 0.81 & 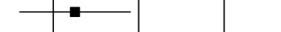 & 6.24 \\
\hline Kuck 2010 & 0.47 & 0.25 & 0.90 & & 33.45 \\
\hline Willems 2020 & 0.62 & 0.38 & 1.01 & & 60.31 \\
\hline & 0.52 & 0.36 & 0.76 & & \\
\hline
\end{tabular}

$\begin{array}{lllll}0.01 & 0.1 & 1 & 10 & 100\end{array}$

Favors Ablation Favors Medical Therapy

Fixed effects mode

Heterogeneity: Tau2 $=0.036 ;$ Chi2=2.511; $d f=2 ; P=0.285 ; 12=20.4 \%$

B

Study name Statistics for each study

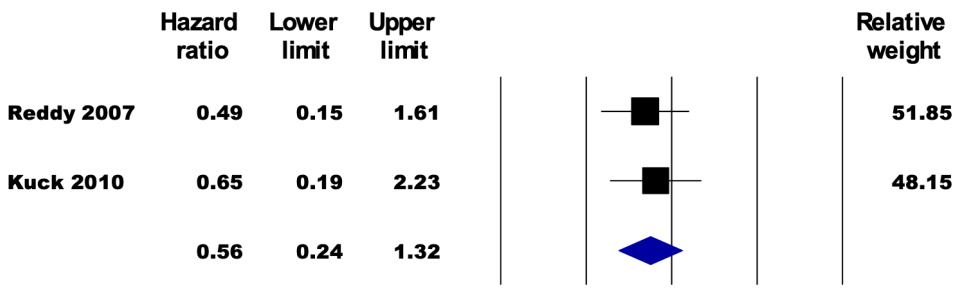

$\begin{array}{lllll}0.01 & 0.1 & 1 & 10 & 100\end{array}$

Favors Ablation Favors Medical Therapy

Fixed effects model

Heterogeneity: Tau $2=0.000 ;$ Chiz $=0.105 ; d f=1 ; P=0.746 ; 12=0.0 \%$

Test for overall effect: $Z=-1.324(P=0.185)$ 
A
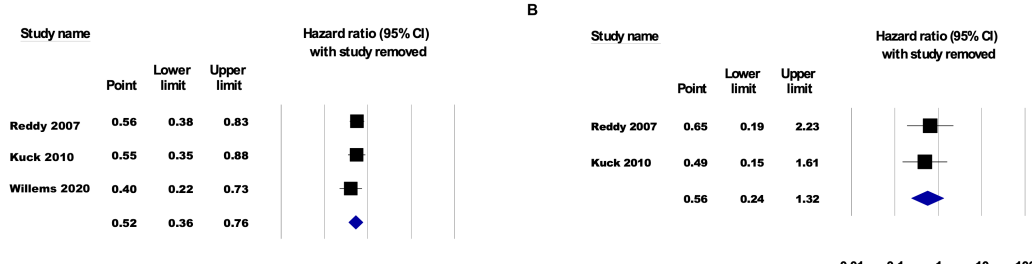

$\begin{array}{lllll}0.01 & 0.1 & 1 & 10 & 100\end{array}$

$\begin{array}{lllll}0.01 & 0.1 & 1 & 10 & 100\end{array}$

Favors Ablation Favors Medical Therapy

Favors Ablation Favors Medical Therapy

c

Study name Cumulative statistics

Lower Upper
Point
limit

$\begin{array}{lllll}\text { Reddy } 2007 & 0.18 & 0.04 & 0.81\end{array}$

$\begin{array}{llll}\text { Kuck } 2010 & 0.40 & 0.22 & 0.73\end{array}$

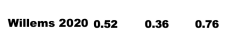

$\begin{array}{lll}0.52 & 0.36 & 0.76\end{array}$

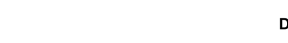

$\begin{array}{lllll}0.01 & 0.1 & 1 & 10 & 100\end{array}$

\begin{tabular}{|r|}
\begin{tabular}{c} 
Relative \\
weight \\
6.24 \\
39.69 \\
100.00 \\
\hline
\end{tabular} \\
\hline
\end{tabular}

D

Favors Ablation Favors Medical Therapy

Study name Cumulative statistics

$\begin{array}{lccc} & \text { Point } & \begin{array}{c}\text { Lower } \\ \text { limit }\end{array} & \text { Upper } \\ \text { limit } \\ \text { Reddy 2007 } & 0.49 & 0.15 & 1.61 \\ \text { Kuck 2010 } & 0.56 & 0.24 & 1.32 \\ & 0.56 & 0.24 & 1.32\end{array}$

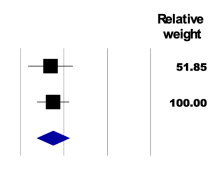

$\begin{array}{lllll}0.01 & 0.1 & 1 & 10 & 100\end{array}$

Favors Ablation Favors Medical Therapy 
A

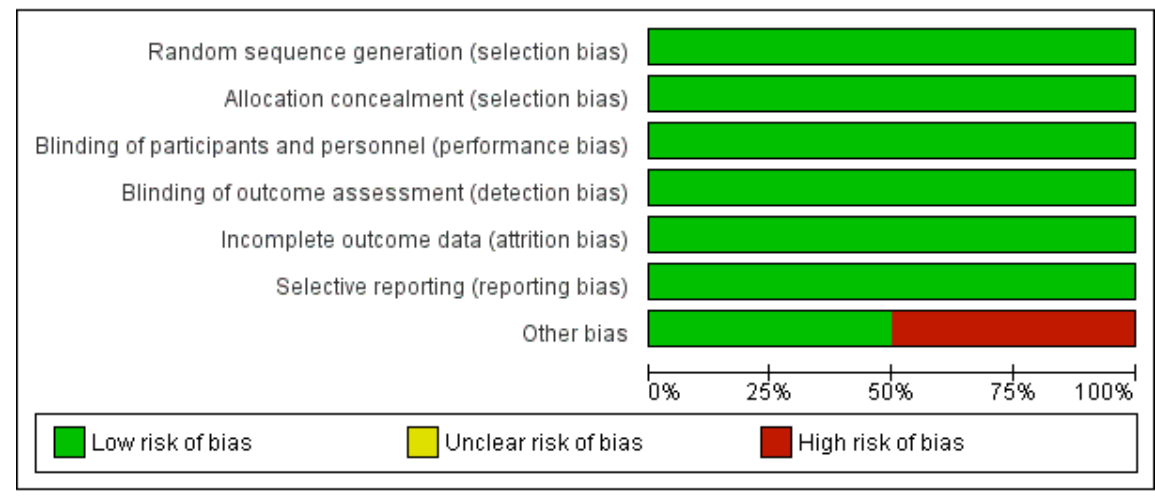

B

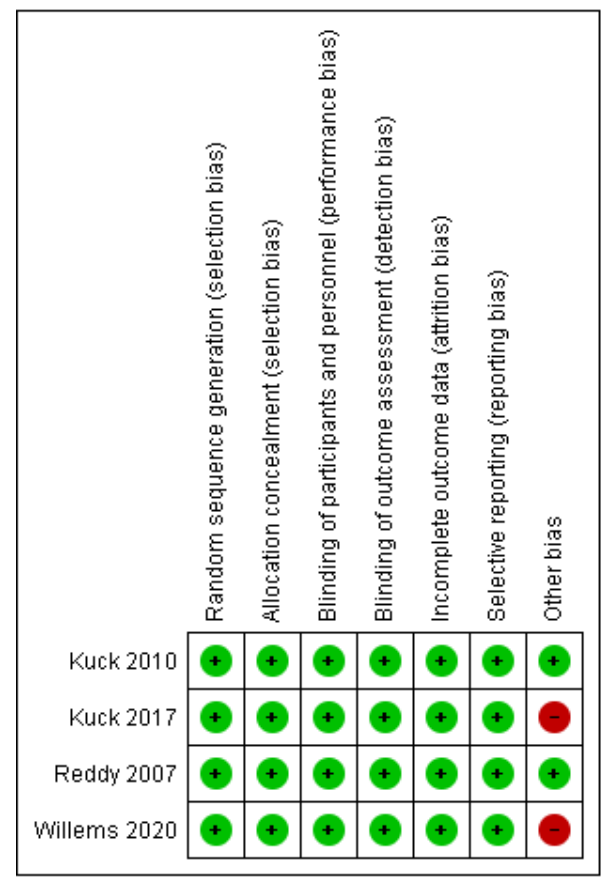

